\section{$\underset{\substack{\text { hommes } \\ \text { \& migrations }}}{ }$}

\section{Hommes \& migrations}

Revue française de référence sur les dynamiques

migratoires

1289 | 2011

Les frontières du sport

\title{
Les associations sportives et folkloriques portugaises
}

L'agglomération bordelaise (1978-2008)

\section{Rémi Solacroup et Fabien Sabatier}

\section{(2) OpenEdition \\ 1 Journals}

\section{Édition électronique}

URL : http://journals.openedition.org/hommesmigrations/797

DOI : 10.4000/hommesmigrations.797

ISSN : 2262-3353

Éditeur

Musée national de l'histoire de l'immigration

Édition imprimée

Date de publication : 1 janvier 2011

Pagination : 62-69

ISSN : $1142-852 X$

Référence électronique

Rémi Solacroup et Fabien Sabatier, «Les associations sportives et folkloriques portugaises », Hommes \& migrations [En ligne], 1289 | 2011, mis en ligne le 31 décembre 2013, consulté le 01 mai 2019. URL : http://journals.openedition.org/hommesmigrations/797 ; DOI : 10.4000/ hommesmigrations.797

Ce document a été généré automatiquement le 1 mai 2019.

Tous droits réservés 


\title{
Les associations sportives et folkloriques portugaises
}

L'agglomération bordelaise (1978-2008)

\author{
Rémi Solacroup et Fabien Sabatier
}

1 Depuis les premiers travaux de l'École de Chicago au début du $\mathrm{xx}^{\mathrm{e}}$ siècle, la recherche scientifique a mis le doigt sur la complexité du processus d'intégration des populations immigrées. Si le phénomène de "dilution" des allochtones dans la société d'accueil fut un temps formalisé par les étapes successives de compétition, de conflit, d'adaptation, puis d'assimilation, les recherches conduites depuis ont attesté les spécificités immigrées en fonction des contextes historiques et territoriaux. Des situations très contrastées caractérisent aujourd'hui le type d'intégration des vagues successives d'immigration et de leur descendance. Si certaines immigrations sont aujourd'hui assimilées, d'autres présentent des profils très divers, parfois négativement nourris par des phénomènes de stigmatisations et de discriminations ou par des facteurs de nature plus culturelle.

2 Les migrations questionnent donc "le vivre ensemble", et le sport associatif est souvent présenté comme une ressource sociale et un dispositif public utiles à l'intégration des immigrés, assertion cependant âprement discutée. C'est pourquoi, depuis quelque temps, l'histoire et la sociologie du sport s'interrogent "sur la place que ce dernier occupe réellement dans nos sociétés et sur les usages qui en [sont] faits" ${ }^{\text {". }}$

3 En prenant l'agglomération bordelaise comme cadre d'analyse et plus particulièrement la ville de Cenon, limitrophe de la métropole régionale, cet article souhaite contribuer à enrichir la somme des travaux déjà produits sur l'immigration en Aquitaine, en s'intéressant plus spécifiquement au rôle joué par le sport chez les immigrations européennes, telle l'immigration portugaise. Les Portugais, souvent qualifiés d'invisibles dans la société française, possèdent, malgré la barrière de la langue, une véritable proximité culturelle avec la France. Issus d'un pays européen, ils sont catholiques, peu marqués phénotypiquement et souvent qualifiés de bien intégrés. Nous savons que cette population migrante a constitué ses premiers clubs sportifs vers le milieu des années soixante. Marqué par une véritable dynamique en un temps où pourtant le droit 
d'association pour les étrangers s'avérait bien restrictif, avant la réintroduction de la liberté d'association en 1981, le tissu sportif associatif portugais en France compte avant 1974 entre cent et deux cents clubs de football.

\section{Le sport, terrain de la défense des identités}

4 Cet engouement sportif répondait à la nécessité d'organiser une sorte de société alternative à une société française perçue comme menaçante. Ainsi, "en France, avec le salariat, [les Portugais] sont confrontés au temps libre [...] pendant lequel ils sentent la nécessité de créer un entre-soi, un espace propre leur permettant de se protéger et de s'adapter progressivement et collectivement à l'espace social français ${ }^{2 ”}$. La forme associative de la pratique, qui aurait pu être informelle, renvoyait pour sa part à la volonté de se confronter, avec une certaine rage de vaincre parfois, aux Français sur le terrain sportif. Le sens réel de cette pratique sociale répondait dans ce premier temps de l'implantation “ à l'entrée dans la modernité, [à une] passerelle d'échange, [une] marchandise de valorisation d'une population se sentant marginalisée et [à] un vecteur d'une revanche symbolique ${ }^{3 "}$.

5 Cette problématique de la "médiation" avec la société d'accueil au cours de ce premier temps de la rencontre (1958-1974), période où l'altérité fut naturellement à son pic, mérite aujourd'hui d'être réévaluée pour les trois longues décennies écoulées depuis. Les premiers immigrants et leurs enfants conservent-ils ou développent-ils toujours aujourd'hui des enclaves associatives communautaires près d'un demi-siècle après la phase principale de migration? Si tel est le cas, ce réseau vise-t-il toujours à reconstruire un Portugal en miniature ou répond-il seulement à l'évitement du "désenchaînement des générations". En somme, existe-t-il aujourd'hui une "portugalité d'intégration", succédant à une "portugalité de médiation"?

\section{L'immigration portugaise en France et à Bordeaux}

6 Au milieu des années soixante, la France découvre soudainement que la période de prospérité économique qu'elle traverse est en partie liée à la présence de nombreux travailleurs étrangers présents sur son territoire. Cependant, lorsque l'on parle d'immigration en France, on évoque rarement les centaines de milliers de Portugais venus en France dès les années soixante. Selon les recensements généraux de la population, le nombre de Portugais présents en France varie à la hausse en passant de 50000 à 760000 individus entre 1962 et 1975 . C'est une immigration économique à laquelle se mêle une immigration politique, composée d'intellectuels ou de jeunes hommes fuyant le régime salazariste mais également la mobilisation pour les conflits coloniaux africains au Mozambique, en Guinée-Bissau ou en Angola. S'installant en majorité dans la région parisienne et dans les grandes métropoles françaises, la présence portugaise en Aquitaine augmente également à partir des années soixante, puisqu'on dénombre à cette période une trentaine de milliers de Portugais.

La ville de Bordeaux est au départ le cœur de l'implantation. Des quartiers de Bordeaux hispanisants comme les quartiers de Saint-Michel ou de Paludate deviennent progressivement des quartiers où se regroupent les immigrés portugais. On dénombre près de 10000 Portugais à Bordeaux en 1982. Une présence lusitanienne massive regroupée dans les mêmes quartiers permet la mise en place progressive d'une véritable 
communauté portugaise développant rapidement un associationnisme sportif. La vie portugaise à Bordeaux connaît ensuite plusieurs implantations au fil des années. En effet, dans un premier temps, les immigrés portugais arrivant à Bordeaux s'installent principalement dans des quartiers populaires à dominante espagnole. On peut y voir deux raisons principales : le coût de l'habitat et la grande proximité culturelle entre Espagnols et Portugais. Cependant, assez rapidement dans les années quatre-vingt, les regroupements familiaux et l'augmentation du pouvoir d'achat des foyers ont permis aux familles portugaises de quitter ces quartiers pour aller vivre dans des banlieues plus salubres comme les communes de Cenon, Villenave-d'Ornon ou Mérignac.

\section{L'associationnisme sportif et culturel portugais en région bordelaise}

8 La création de plusieurs associations sportives ou culturelles affichant ouvertement leur facture portugaise atteste d'une dynamique autour du Portugal, à l'instar des associations telles 0 Sol de Portugal et le Football-Club Portugais. Un relevé d'associations en Gironde montre que 43 associations sportives ayant dans leur intitulé les noms ou les mots "Portugal", "Portugais", "Lusitain" ou "Lusitanos" ont été constituées depuis la phase principale d'immigration. Généralement, le mot "Portugal" est évoqué dans le nom de l'association, et parfois par d'autres symboles telles les couleurs vertes et rouges des maillots ; les associations d'obédience portugaise sont avant tout le lieu de retrouvailles, de partage, mais également un lieu de revendication publique d'une double appartenance nationale et parfois culturelle. Le dynamisme et la pérennité de ces associations fluctuent, mais le flux de création ne s'est pas tari, bien au contraire, puisque des associations portugaises se créent toujours aujourd'hui. Le premier type d'association est strictement sportif et se caractérise par la présence de plusieurs clubs de football, tels le Football-Club Portugais ou l'AS Lusitanos de Cenon. Le second type est plutôt culturel : ce sont des groupes de danses folkloriques portugaises comme l'association Alegria de Cenon. Ces deux profils associatifs renvoient en somme à deux registres distincts de la "portugalité".

\section{Les danses traditionnelles, premier ciment de la communauté portugaise aujourd'hui}

9 Plusieurs groupes de danses traditionnelles portugaises se proposent de constituer des troupes de danseurs pratiquant différentes danses régionales portugaises. On dénombre une dizaine de ces formations de nos jours dans la région bordelaise.

Pour exemple, Alegria, fondée en 1982, affiche son ambition de diffuser la culture folklorique du pays et d'enrayer le déclin de l'usage de la langue portugaise au sein de la seconde génération. Pour la génération ayant migré il fallait impérativement promouvoir la transmission du patrimoine national. Cette association a ainsi baigné dès sa création dans la culture lusitanienne. La principale langue parlée a toujours été le portugais et le siège de l'association est finalement progressivement devenu un lieu de regroupement pour une partie de la communauté, mais également un lieu pour célébrer différentes fêtes du Portugal. D'autres groupes de danses traditionnelles sont apparus au fil des ans autour de Bordeaux, voire dans la Gironde, avec cette même idée directrice de transmission du patrimoine culturel aux nouvelles générations. Ces associations culturelles, par le biais de 
nombreux défilés lors de festivals partiellement consacrés à la culture portugaise, ont finalement conquis la scène publique bordelaise.

11 On remarque donc que ces groupes de danses traditionnelles sont des lieux privilégiés de "portugalité" dans le sens où la culture portugaise est le moteur principal de ces associations. Par ailleurs, comme pour le football, l'espace associatif est le lieu d'une fierté des origines, du besoin de préserver une forme de communauté portugaise et plus rarement d'afficher une visibilité sociale. L'implication de la troisième génération dans ces associations résulte d'une volonté d'honorer, pour certains adolescents ou jeunes adultes, la culture portugaise : "J'aime bien danser à Alegria, on est un groupe d'amis et on se retrouve. Je suis fier d'être d'origine portugaise et c'est pour cela que je danse ici. [...] Je fais du football au club de Lormont car même si je suis fier d'être portugais, je suis fier d'être aussi d'ici. C'est important pour moi d'avoir les deux identités ${ }^{4}$." Cette insertion dans le tissu associatif portugais se combine également avec la pratique des nouvelles cultures urbaines comme le hip-hop. Cette jeunesse d'origine portugaise présente manifestement un attachement au Portugal, mais combiné au sentiment bien marqué d'être française.

\section{Le football associatif dans la périphérie bordelaise au cours des dernières décennies}

12 Le changement d'implantation des Portugais du centre vers la périphérie bordelaise provoque un transfert de leur milieu associatif vers les nouvelles communes périurbaines et implique des changements de statuts, des dissolutions et la création de nouvelles associations. Par exemple, citons ici le Club Sportif Portugais (CSP) situé aujourd'hui à Villenave-d'Ornon, une commune proche de Bordeaux qui a connu plusieurs sièges dans la communauté urbaine de Bordeaux. Cela atteste le souci de conservation ou de renouvellement d'un tissu associatif portugais après la première phase d'insertion en région bordelaise, probablement accentué par la faible dispersion de l'immigration portugaise dans les nouveaux territoires d'implantation.

Dans le cas girondin, les clubs de football qui revendiquent l'appartenance au Portugal sont aujourd'hui au nombre de deux dans la banlieue bordelaise. D'autres clubs ont existé puis ont disparu comme le Football-Club Portugais, créé dans les années soixante, puis dissous dans les années quatre-vingt. Remarquons ici que nous parlons bien de clubs entièrement portugais et non d'équipes "portugaises" au sein d'un club sans revendi cation nationale particulière. Il existe donc aujourd'hui le Club Sportif Portugais (CSP) à Villenave-d'Ornon et l'AS Lusitanos (ASL) de Cenon. Le choix du football s'est imposé comme une évidence, comme le signale un membre fondateur : "Le football, tout le monde aime ça, et c'est un sport très populaire au Portugal comme en France. Lorsque le club a été créé par les Portugais, tous [étaient] des anciens joueurs qui jouaient au foot là-bas. Ils ne se sont pas posé la question du sport. Le football était une évidence. En plus, ce n'était pas trop compliqué d'y jouer." Cette réponse rend bien compte d'un discours déjà présent lors de la période précédente, celle d'une culture "foot" importée du pays d'émigration, d'autant plus accentué que le football est déjà bien implanté en France.

Le CSP est créé en 1978 par de jeunes immigrés portugais afin de regrouper les joueurs de football de la communauté portugaise. Il n'existait aucune revendication particulière à sa création, si ce n'est le souhait d'afficher une appartenance nationale et l'envie de se réunir entre Portugais autour de la passion du football. Très rapidement, ce club a su 
attirer des licenciés portugais ou d'origine portugaise, d'abord du quartier, puis de la ville de Bordeaux, et enfin de la communauté urbaine de Bordeaux. Cependant, dans les années quatre-vingt et quatre-vingt-dix, le CSP s'est heurté à la logique compétitive du sport. Il lui fallait posséder la meilleure équipe sportive afin d'obtenir des moyens financiers pour la vie du club, imposant de remettre en cause la majorité lusitanienne des joueurs dans l'équipe. Par ailleurs, le club se retrouvait également confronté au problème d'une "hémorragie" de ses meilleurs joueurs d'origine portugaise souvent amenés à jouer dans des clubs de divisions supérieures. Le club a donc dû faire progressivement appel à des licenciés d'origine non portugaise pour poursuivre l'aventure. Aujourd'hui, même si la majorité des joueurs du club et des dirigeants sont d'origine portugaise, une partie non négligeable d'entre eux ne vient pas "du pays".

La création de l'AS Lusitanos de Cenon en 2002 répond au même vœu de réunir les joueurs issus de la communauté portugaise autour du football. Cependant, beaucoup plus rapidement que le CSP, l'ASL s'est heurtée à l'exigence d'une culture sportive fondée sur la performance au détriment partiel d'une identité spécifiquement portugaise. Aujourd'hui, si l'équipe dirigeante reste strictement d'origine portugaise, les licenciés qui composent les équipes seniors et jeunes sont issus de différentes composantes de la mosaïque France, dans une commune particulièrement marquée par son cosmopolitisme. En outre, si le portugais fut initialement la langue "officielle" au sein du club, il n'en est plus rien aujourd'hui, le français l'ayant largement remplacé. Néanmoins, ces deux clubs de football représentent un vecteur important de formation de la communauté portugaise de Bordeaux puisque les rencontres ou les tournois drainent un public d'origine portugaise. Tous les week-ends, le stade devient le lieu de célébration d'un entre-soi portugais.

\section{Une autre portugalité sportive?}

Le football et le folklore portugais à Bordeaux sont bien une réalité. Plusieurs associations regroupent plusieurs centaines d'adhérents et proposent à la communauté portugaise la possibilité de jouer ou de revendiquer une appartenance particulière. Les clubs de football répondent aujourd'hui aux principes qui règlent la vie sportive compétitive puisque la vocation culturelle et nationale de ce tissu associatif est sensiblement transformée par les lois du sport favorisant le résultat sportif. Ainsi, le départ de joueurs d'origine portugaise des clubs portugais, l'arrivée de joueurs sans histoire commune avec le Portugal, l'abandon de la langue comme signe premier de portugalité traduisent probablement la fin d'une vision communautaire défensive telle qu'elle a pu se concevoir dans la période précédente. Pour les associations folkloriques, l'ancrage portugais est manifestement plus marqué, mais la jeunesse ne conçoit les activités proposées que sous l'angle de l'appoint aux pratiques sociales dénuées de toutes références aux origines. La culture et le sport représentent non plus un point de friction avec "les Français", mais une simple frontière, intermittente et particulièrement poreuse. Une partie de la jeune génération reste attachée au Portugal, mais sans traduire dans son engagement une tension particulière avec la société française, et plutôt comme l'expression d'une double appartenance bien vécue. Cette jeunesse s'implique dans diverses associations sans lien avec le Portugal comme le signale Léa, jeune Française d'origine portugaise: "Je fais également du hip-hop en dehors du groupe folklorique. [...] J'ai des amis qui font du hand-ball ou de la gymnastique. On est beaucoup dans ce cas-là." 
17 À nos yeux, ces nouvelles modalités communautaires émanent de l'intégration réussie des enfants de cette immigration, mais attestent pour certains d'entre eux un refus de l'abandon de traits culturels propres au pays d'origine. Le folklore et le sport offrent une nouvelle façon d'être français qui permet un lien intergénérationnel initié par les anciens, mais aujourd'hui relayé par la seconde génération.

\section{Conclusion}

À Bordeaux et dans sa périphérie, il existe toujours aujourd'hui un associationnisme portugais. Le souci de la transmission d'un patrimoine culturel et du sentiment d'appartenance nationale reste présent dans une partie de la seconde et de la troisième générations. Les danses folkloriques et le sport jouent un rôle prépondérant dans la formation de cette "portugalité d'intégration". Par ailleurs, cet associationnisme permet la réunion des générations, dans une ambiance pour l'essentiel festive au cours des weekends. Ce positionnement particulier des nouvelles générations correspond globalement aux conclusions des dernières enquêtes de l'Insee-Ined sur l'immigration en France et sa descendance. La jeunesse française d'origine portugaise présente de bons indicateurs d'intégration, mais pour une partie d'entre elle, dans la conservation d'une relative proximité avec le Portugal, l'attachement à une mémoire des origines, probablement indispensable à une vie sociale équilibrée et à l'évitement d'un désenchaînement irréversible des générations.

Enfin, il convient de fixer une limite à cette étude, le tissu associatif dédié au folklore et au football draine quelques centaines de personnes tout au plus, pour une immigration et une descendance probablement proches d'une trentaine de milliers d'individus en région bordelaise. Cette assertion laisse à penser que pour le cas des Portugais de France et de leurs enfants, les modalités d'intégration suivent ces étapes annoncées en introduction. Mais il semble probable que le dernier temps de l'assimilation complète, telle qu'elle a pu se produire en grande partie pour les Italiens par exemple, ne reflète pas l'avenir pour les "Portugais" dans une société française où le pluriculturalisme est aujourd'hui socialement accepté amplement dans la mesure où il ne déroge pas à certains principes républicains.

\section{NOTES}

1. Viévard Ludovic, Le sport : outil d'intégration et de mixité?, Rapport pour le centre de ressources de la DPSA, Grand Lyon, 2006, p. 4.

2. Pereira Victor, "Le football parmi les migrants portugais en France, 1958-1974", in Migrance, Yvan Gastaut (dir.) "Sport et immigration : parcours individuels, histoires collectives", $\mathrm{n}^{\circ} 22$, 2003, p. 32.

3. Pereira Victor, art. cit., p. 38.

4. Entretien avec Julien, jeune Français d'origine portugaise, 17 ans, le 16 mai 2009. La teneur de ses propos présente une certaine récurrence avec les différents entretiens réalisés. 


\section{RÉSUMÉS}

Dans la mosaïque de cultures qui constituent la France contemporaine, la pratique sportive et le folklore demeurent des marqueurs identitaires forts. Depuis leur installation à Bordeaux et dans sa périphérie dès les années soixante, les Portugais se sont fédérés en associations sportives et culturelles. L'objectif était tout autant de maintenir le lien avec le pays d'origine que de créer les conditions d'un entre-soi dans le champ des loisirs. Aujourd'hui, ce tissu associatif demeure et engage les nouvelles générations à poursuivre la transmission de la culture lusitanienne.

\section{AUTEURS}

\section{RÉMI SOLACROUP}

Doctorant

\section{FABIEN SABATIER}

MCF, université Victor-Segalen, Bordeaux-2, LACES (Laboratoire cultures, éducation, sociétés) 